\title{
Close Encounters between AMC and MoviePass
}

\author{
Debabrata Dey \\ Foster School of Business \\ University of Washington \\ ddey@uw.edu
}

\author{
Atanu Lahiri \\ Jindal School of Management \\ University of Texas at Dallas \\ atanu.lahiri@utdallas.edu
}

\author{
Rajiv Mukherjee \\ Cox School of Business \\ Southern Methodist University \\ mukherjee@mail.smu.edu
}

\begin{abstract}
Two threads regarding the recent feud between $A M C$ Theaters and MoviePass have been quite popular in the news cycle: (i) the impending bankruptcy of MoviePass and (ii) the Twitter war between the two players. In this paper, we seek to evaluate if such developments are mere aberrations or if there is any economic rationale behind them. We develop a parsimonious microeconomic model for this purpose that sheds light on the competition/cooperation responses of a theater chain towards the entry of a non-essential intermediary. Our results have important implications for management and policy.

Keywords: Intermediary, platform, competition, subscription, theater, motion picture
\end{abstract}

\section{Introduction}

In recent times, the name MoviePass has been making frequent rounds in the headlines. In particular, two threads have been quite popular: (i) the impending bankruptcy that MoviePass faces from "burning" its money [8], and (ii) the fierce war of words that broke out in the Twitter sphere between MoviePass and AMC Theaters [2]. As observers, we found both developments interesting.

First, MoviePass's impending bankruptcy predictions seemed questionable. After all, just a few months earlier, the majority stake in the company had been purchased by a New-York-based data analytics firm, Helios and Matheson Analytics, or HMA in short. The "money-burning" approach that MoviePass had undertaken was implemented well before its acquisition by HMA. Now, could $H M A$ actually be this naive in their investment decisions? Had their investment calculus not considered the full import of MoviePass' money-burning strategy? Second, quite remarkable was the sudden hostile posture that MoviePass and AMC seemed to have taken against each other despite their initial partnership in 2014. What could have transpired between the two that would so suddenly break their seemingly thriving business alliance? In December of 2014, in an URI: https://hdl.handle.net/10125/64477

978-0-9981331-3-3

(CC BY-NC-ND 4.0) interview with the New York Times, Christina Sternberg, the senior vice president for corporate strategy at AMC, had defended the collaboration with MoviePass by saying, "It frankly wouldn't be smart to ignore the success of subscription in other areas of media." If the idea of a third-party subscription platform was this critical then, what could have changed that perception so soon, in less than three years?

We found these questions quite intriguing and decided to put them under a microscope with a microeconomic lens. The purpose of this research, therefore, is to seek economic intuitions behind such surprising observations in practice. In particular, we want to study the game-theoretic interactions between two companies - a near-monopolist retailer (AMC) and a "non-essential" intermediary (MoviePass) - to better understand their "hot-andcold behavior" of cooperation and competition, and to examine if the recent developments and predictions have any economic basis.

Some context is perhaps necessary here. Since the severe economic downturn in 2008-09, the annual revenue of the US and Canada box office has been hovering around the $\$ 10$ to $\$ 11$ billion mark and has failed to put together any consistent upward trend over the last decade. In fact, the three major movie-theater chains in the US and CanadaAMC, Regal, and Cinemark - have all been struggling to attract moviegoers. It was into this stagnated industry that MoviePass made its first entry in 2011. Before its entry, the industry consisted of a three-tiered-studio (manufacturer) to distributor to theater/exhibitor (retailer) - supply chain, with significant monopolistic power at each level [17]. With its smartphone-based subscription platform, ${ }^{1}$ MoviePass wanted to insert itself into this supply

${ }^{1}$ The entire MoviePass model is administered through a smartphone making use of its internet and GPS connections. At the time of signing up, every subscriber is issued a debit card. When a subscriber is in close proximity of a particular theater, he can find out which movies are available at that theater at that time. Now, if he chooses to view one from this 
chain as a fourth player, between a theater and its consumers (the moviegoers), working in tandem with the existing channel (direct sales). While only a few small/fringe theater showed interest in MoviePass intitially, by 2014, AMC entered into a partnership with MoviePass [6]. In 2017, MoviePass made a "mad" dash to increase its subscription base by announcing a subscription fee of only $\$ 9.95$ to watch upto 30 movies a month, an amount that was hardly enoough to cover an individual ticket. All major theater chains, with AMC leading the pack, criticized this move. And, almost magically, within a short time thereafter, all these chains rolled out their own platform/app and announced their own subscription services.

There are several streams of literature related to this work. Among them is the literature on intermediation. Intermediaries are firms that connect buyers with sellers and generate value by reducing transaction costs [5], which include search, fit, and coordination costs as well as risks arising from information asymmetry [7, 9]. Prior research has diligently studied how a market may evolve through intermediation, disintermediation, and reintermediation $[4,10]$. We do not study this process; instead, we contribute by studying whether or not a seller should combat a nonessential intermediary that seeks to insert itself between the seller (a movie theater) and buyers.

Even though a pass that allows a consumer to watch multiple movies is akin to a bundle of movies [1] — or perhaps a quasi-bundle as the choice of the bundle constituents is in the hands of the consumer [11] - our objective in this paper is quite a bit different from that of the traditional bundling literature. First, we do not focus on the issue of reducing demand elasticity through bundling. Second, when a pass is offered to consumers along with individual tickets, there would be usage-based discrimination [18]. Unlike prior research, however, both our seller and the intermediary can offer a pass, which leads to interesting strategic dilemmas. Specifically, (i) should the intermediary target only frequent consumers or all consumers, and (ii) should the theater cooperate with the intermediary or compete against it by offering its own subscription service? We are among the first to carefully study these questions.

set, the amount of money necessary to buy an individual ticket at the counter/kiosk would be immediately transferred to his debit card, which he can use to purchase the ticket. This business model guarantees that a theater can never really block a MoviePass subscriber from watching movies at its facilities and MoviePass does not need a prior contract to invade a market.

\section{Model Preliminaries}

We start with a situation where there is no intermediary, but there is just a monopolist movie theater who sets the price, $p>0$, at which consumers (moviegoers) are offered individual movie tickets. Also, the theater has the option of offering a periodic subscription plan-henceforth, called a passat a fee, $f>0$, which would allow a subscriber to see all the $n$ movies released during the subscription period; in our model, $n$ is exogenous. Facing such a pricing scheme, our moviegoers make their actual consumption decisions.

\subsection{Consumer Behavior}

We consider a heterogeneous set of moviegoers whose mass is normalized to one. Our moviegoers belong to two broad categories based on their proclivity towards watching movies in a theater: (i) high type: a $\lambda$ fraction of frequent moviegoers who regularly visit the theater, and (ii) low type: the remaining $(1-\lambda)$ fraction of infrequent moviegoers who are occasional visitors to the theater. Consumers are also heterogeneous in their taste for movies; different consumers derive different values from watching a movie and, in addition to their own proclivity towards watching movies. Clearly, each movie $j$ is a different experience for a moviegoer $i$, and each moviegoer gets a different benefit from watching it. Therefore, we assume:

Assumption 1. A frequent moviegoer $i$ 's valuation for a movie $j, j \in\{1,2, \ldots, n\}$, is $v_{i j}$, whereas that of an infrequent moviegoer is $\left(v_{i j}-\mu\right), v_{i j}$ i.i.d. and uniform over $[0,1]$ and $\mu \in(0,1)$ a constant.

In essence, Assumption 1 tells us that for any given movie $j$, consumer valuations are i.i.d. and drawn from a uniform distribution over $[0,1]$ and, at the same time, for any consumer $i$, his valuations for all $n$ movies are also i.i.d. and drawn from the same distribution. One can view $\mu$ as a parameter that captures the added inertia or lack of motivation faced by infrequent moviegoers.

Frequent Moviegoers. If a high type buys the pass at $f$, he would surely want to derive the maximum benefit from it; that is, he would end up seeing all the $n$ movies allowed by the pass. Since his valuation for each of these $n$ movies is uniform over $[0,1]$, his expected valuation for each movie is onehalf, implying that the following individual rationality constraint must be satisfied for him to buy the pass: $\frac{n}{2}-f \geq 0$.

To derive his incentive compatibility constraint for the pass, we consider his overall benefit from buying 
individual tickets instead. First, the frequent consumer $i$ watches a movie $j$ iff $v_{i j}-p \geq 0$; he refrains otherwise. Therefore, his expected surplus from tickets per subscription period is $\frac{n(1-p)^{2}}{2}$. As long as this surplus is below the surplus from the pass, $\frac{n}{2}-f$ that is, as long as $f \leq n p\left(1-\frac{p}{2}\right)$-he would prefer the pass over purchasing individual tickets.

Infrequent Moviegoers. Unlike the high type, a low type would not see all the $n$ movies even if he buys the pass. Only a $(1-\mu)$ fraction of movies would satisfy: $v_{i j}-\mu \geq 0$. So, his expected surplus per period is $\frac{n(1-\mu)^{2}}{2}$, which must be no less that $f$ for him to buy the pass. Now, an infrequent consumer $i$ who does not have the pass would buy a ticket to watch movie $j$ iff $v_{i j}-p-\mu \geq 0$; accordingly, his expected per-period surplus from tickets would be $\frac{n(1-p-\mu)^{2}}{2}$. When this surplus is no bigger than the surplus from tickets - that is, $f \leq n p\left(1-\mu-\frac{p}{2}\right)$ - he would prefer the pass over individual tickets.

\subsection{Theater's Problem}

Typically, studios and distributors charge the theater a pre-negotiated amount per seat sold [14], so we assume that the theater faces a marginal cost of $c$ per sold seat:

Assumption 2. A theater incurs a marginal cost $c>0$ for each theater seat sold.

If the theater offers a subscription plan, it must choose $p$ and $f$ in such a way that a consumer's appropriate IR and IC conditions are met. For a given $p$, the theater's profit is increasing in $f$. Therefore, the theater would choose as high an $f$ as possible that barely satisfies one of these constraints, making the appropriate constraint an equality in equilibrium. Hence, we have:

LEMMA 1. The theater sells the pass at $f^{*}=$ $\frac{n p(2-p)}{2}$ if it wants only the frequent consumers to subscribe to it. If the theater wants to attract infrequent consumers as well, it sets $f^{*}=\frac{n p(2(1-\mu)-p)}{2}<$ $\frac{n p(2-p)}{2}$.

It is clear from Lemma 1 that, it is impossible for the theater to price in a way that attracts only the low type and keeps the high type out. Therefore, three broad scenarios are possible for the theater:

Case 1: Sell Tickets Only, No Pass. When the pass is not offered, for every movie, a $(1-p)$ fraction of the high type and a $(1-p-\mu)$ fraction of the low type shall buy a ticket. The total demand faced by the theater from all the $n$ movies is, therefore, given by:

$$
q= \begin{cases}n \lambda(1-p)+n(1-\lambda)(1-p-\mu), & \text { if } p<1-\mu \\ n \lambda(1-p), & \text { otherwise }\end{cases}
$$

where the second case, $p \geq 1-\mu$, represents a situation in which the low types are priced out of the market and only the high types buy tickets; this limiting subcase of Case 1 is denoted as Case 1L. Maximizing the theater's profit, $\pi=q(p-c)$, we get:

Proposition 1. When selling only tickets, the theater's optimal price and profit are:

$$
\begin{aligned}
& p^{*}= \begin{cases}p_{1}^{*}=\frac{1+c-\mu(1-\lambda)}{2}, & \text { if } \lambda<\left(\frac{1-c-\mu}{\mu}\right)^{2}, \\
p_{1 \mathrm{~L}}^{*}=\frac{1+c}{2}, & \text { otherwise, }\end{cases} \\
& \pi^{*}= \begin{cases}\pi_{1}^{*}=\frac{n(1-c-\mu(1-\lambda))^{2}}{4}, & \text { if } \lambda<\left(\frac{1-c-\mu}{\mu}\right)^{2}, \\
\pi_{1 \mathrm{~L}}^{*}=\frac{n \lambda(1-c)^{2}}{4}, & \text { otherwise. }\end{cases}
\end{aligned}
$$

Case 2: Sell Pass to Only Frequent Consumers. In this case, the theater sets $f^{*}=\frac{n p(2-p)}{2}$, as per Lemma 1. Since those who subscribe to the pass watch all the $n$ movies, the total profit from the subscription plan is $\lambda\left(f^{*}-n c\right)$. Also, as long as $p<1-\mu$, the theater enjoys some additional profit from ticket sales to the low types. The overall profit to the theater can then be written as:

$$
\pi= \begin{cases}\lambda\left(\frac{n p(2-p)}{2}-n c\right)+ & \\ n(p-c)(1-\lambda)(1-p-\mu), & \text { if } p<1-\mu \\ \lambda\left(\frac{n p(2-p)}{2}-n c\right), & \text { otherwise. }\end{cases}
$$

As before, the second case $(p \geq 1-\mu)$ arises when the low types are priced out of the market; this subcase of Case 2 is denoted Case 2L. Maximizing the theater's profit, we find the equilibrium solution:

Proposition 2. When selling passes to only the frequent consumers, the theater's optimal tariffs are:

$$
\left(p^{*}, f^{*}\right)= \begin{cases}\left(p_{2}^{*}, f_{2}^{*}\right), & \text { if } \lambda<\frac{(1-c-\mu)^{2}}{(1-c)^{2}+\mu^{2}} \\ \left(p_{2 \mathrm{~L}}^{*}, f_{2 \mathrm{~L}}^{*}\right)=\left(1, \frac{n}{2}\right), & \text { otherwise }\end{cases}
$$

where $p_{2}^{*}=\frac{1+(1-\lambda)(c-\mu)}{2-\lambda}$ and

$$
f_{2}^{*}=\frac{n(1+(1-\lambda)(c-\mu))(3-2 \lambda-(1-\lambda)(c-\mu))}{2(2-\lambda)^{2}} .
$$

Correspondingly, the theater's optimal profit is given by: $\pi_{2}^{*}=\frac{n\left(1+\left(c^{2}-\mu^{2}\right)(1-\lambda)^{2}-2(1-\mu(1-\lambda))(c+\mu(1-\lambda))\right)}{2(2-\lambda)}$ and $\pi_{2 \mathrm{~L}}^{*}=\frac{n \lambda(1-2 c)}{2}$. 
Case 3: Sell Pass to All Consumers. In this case, from Lemma 1 , the theater sets $f^{*}=$ $\frac{n p(2(1-\mu)-p)}{2}$, and all consumers subscribe to the pass. Among them, the high types watch all the $n$ movies, but the low, only $n(1-\mu)$. Therefore, the total profit from the subscription plan is $\pi=\lambda\left(f^{*}-n c\right)+(1-$ $\lambda)\left(f^{*}-n c(1-\mu)\right)$. Individual tickets, although still sold nominally, are no longer purchased; so, they do not contribute to the overall profit. We maximize the theater's profit to get:

Proposition 3. When selling passes to all the consumers, the theater's optimal tariffs are: $p_{3}^{*}=1-$ $\mu$ and $f_{3}^{*}=\frac{n(1-\mu)^{2}}{2}$. The theater's optimal profit is $\pi_{3}^{*}=\frac{n((1-\mu)(1-\mu-2 c)-2 c \lambda \mu)}{2}$.

\subsection{Theater's Optimal Strategy}

It is clear from the earlier analyses that the theater can adopt any one of the above three strategies. Naturally, it would choose the strategy that yields the highest profit for a given set of parameter values. Since closed-form profit expressions exist in each of the five cases - Cases 1, 1L, 2, 2L, and 3-it is easy to analytically compare them in a pairwise fashion to ascertain the possible boundaries that separate these cases. Fortunately, closed-form expressions exist for these boundaries, too, and the entire parameter space can be partitioned into five regions, one each for the five cases:

TheOrem 1. Let $\gamma_{1}=1-\mu(1+\sqrt{\lambda}), \gamma_{2}=$ $\sqrt{2}-1, \quad \gamma_{3}=\frac{1-\mu(1-\lambda)}{1+\sqrt{2(2-\lambda)}}, \quad \gamma_{4}=(1-\mu)(\sqrt{2}-1)-$ $\mu \lambda, \quad \gamma_{5}=\frac{(1-\mu)^{2}-\lambda}{2(1-\mu)(1-\lambda)}, \gamma_{6}=1-\frac{\mu(1+\sqrt{\lambda(2-\lambda)})}{1-\lambda}$, and $\gamma_{7}=\frac{(1-\mu)(\sqrt{2-\lambda}-1)-\mu \lambda}{1-\lambda}$. Furthermore, for all $\lambda \in$ $\left[\frac{1-2 \mu}{2(1-\mu)}, 1\right]$, define $\gamma_{8}=\xi_{1}+\sqrt{\xi_{2}}$ and $\gamma_{8}^{\prime}=\xi_{1}-\sqrt{\xi_{2}}$, where $\xi_{1}=1-2 \lambda-\mu(1-\lambda)$ and $\xi_{2}=2 \lambda(2 \mu(1-$ $\lambda)-1+2 \lambda)$. Then, the following equilibrium regions emerge:

- Region 1: Ticket to All. The theater sells individual tickets to both consumer segments if (i) $\gamma_{3}, \gamma_{4} \leq c<\gamma_{1}$, and (ii) $c \geq \gamma_{8}$ or $c<\gamma_{8}^{\prime}$.

- Region 1L: Ticket to High. The theater sells individual tickets to the high type and prices out the low type if $c \geq \gamma_{1}, \gamma_{2}$.

- Region 2: Pass to High, Ticket to Low. The theater sells the pass to the high type and individual tickets to the low if $\gamma_{5} \leq c<\gamma_{3}, \gamma_{6}$.

- Region 2L: Pass to High. The theater offers the pass to the high type and prices out the low if (i) $\gamma_{6}, \gamma_{7} \leq c<\gamma_{2}$, and either (ii) $\gamma_{8}^{\prime} \leq c<\gamma_{8}$ or (iii) $c \geq 1-\mu$.

- Region 3: Pass to All. The theater offers the pass to both consumer segments if $c<\gamma_{4}, \gamma_{5}, \gamma_{7}$.

\section{Entry of a Digital Platform and the Ensuing "Competition"}

Having described its pricing strategy as a monopoly, we now consider how the theater would respond when a digital platform (MoviePass) enters the market and offers a subscription service for $n$ movies at a fee of $g$ per subscription period. Such a pass provides its subscribers not only the benefit of watching all $n$ movies, but also the flexibility to watch them at other theaters that may be more convenient at times. We capture this flexibility - as well as any other cross-consumption benefits a third-party platform may provide - by ascribing to the pass an additional utility of $\delta \in(0,1)$ per movie, that is, a total of $\delta n$ for each high type and $\delta n(1-\mu)$ for each low type.

The platform incurs a marginal cost, say $r$, per seat; that is, $r$ is the per-seat transfer price from the platform to the theater. Since the platform can always purchase tickets for its subscribers at the market price $p$, it has no incentive to pay any higher. Therefore, $r$ cannot be any higher than $p$, the price charged by the theater for an individual ticket. Furthermore, the theater's profit is always increasing in $r$, so the theater would like to set $r$ as high as possible. Therefore:

LEMMA 2. In the subgame perfect equilibrium, $r=p$, where $p$ is the price charged by the theater for an individual ticket.

Just like the theater, the platform also lacks the ability to price out the high type while attracting the low type. Therefore, the platform has three choices: (i) do not enter the market, (ii) offer the pass to only the high type, or (iii) offer the pass to all consumers. When offering the pass only to the high type at fee $g$, the platform must ensure that the high type would actually purchase it, implying $\frac{n}{2}+\delta n-g \geq \frac{n(1-p)^{2}}{2}$, or equivalently, $g \leq n\left(\delta+p\left(1-\frac{p}{2}\right)\right)$.

When offering the pass to all consumers, however, the platform must ensure that the low type also participates. This it can do by choosing a $g$ that abides by $\frac{n(1-\mu)^{2}}{2}+\delta n(1-\mu)-g \geq \frac{n(1-p-\mu)^{2}}{2}$, which is equivalent to $g \leq n\left(\delta(1-\mu)+p\left(1-\mu-\frac{p}{2}\right)\right)$. Since the platform's profit is linearly increasing $g$, it would choose the maximum possible value while ensuring participation from consumers, as described above. In other words:

LEMMA 3. In the subgame perfect equilibrium, the platform sets the pass at $g^{*}=n\left(\delta+\frac{p(2-p)}{2}\right)$ if it wants only the frequent consumers to subscribe to it. On the other hand, if the platform wants to attract infrequent consumers, it sets $g^{*}=$ 
$n\left(\delta(1-\mu)+\frac{p(2(1-\mu)-p)}{2}\right)$, a lower subscription fee that lures the frequent segment in as well.

Now, when offering the pass only to the high type at $g$, the platform makes a total profit of $\pi_{\text {plat }}=$ $\lambda\left(g^{*}-n p\right)$. It would offer such a pass only if this profit is positive, implying $g^{*} \geq n p$. On the other hand, if it offers the pass to all consumers, the platform would make a total profit of:

$$
\pi_{\text {plat }}=\lambda\left(g^{*}-n p\right)+(1-\lambda)\left(g^{*}-n p(1-\mu)\right) .
$$

For this profit to be positive, we must have $g^{*} \geq$ $\lambda n p+(1-\lambda) n p(1-\mu)$. The next result then follows directly from Lemma 3 :

THEOREM 2. The platform would offer the pass to the high type only if $\delta \geq \frac{p^{2}}{2}$, and to both types only if $\delta \geq \frac{p(p+2 \lambda \mu)}{2(1-\mu)}$. Equivalently, the theater can block the platform's market entry by setting $p>\sqrt{2 \delta}$.

The entry deterrence mechanism in Theorem 2 is markedly different from the typical ones found in the economics literature. In the literature, the incumbent firm usually sets the price so low that potential entrants are discouraged from entering [19, p.306]; in contrast, in Theorem 2, the incumbent actually raises the price to stave off the entrant. There is one similarity, though. In both cases, the entry deterrence turns out to be a costly proposition to the incumbent. Just as a lower price can lead to a lower revenue, so can a higher price by shrinking demand to a suboptimal level.

Thus, our theater holds a costly option of deterring entry by choosing a price higher than $\sqrt{2 \delta}$. Of course, it would exercise that option only when it is actually more profitable to do so. Now, if it does exercise, that is, if $p>\sqrt{2 \delta}$, our solutions in the previous section-see Propositions 1, 2, and 3-would apply as before. Therefore, we now focus on the case where $\delta$ is sufficiently large and the theater and the platform coexist in the market.

\subsection{Theater's Problem in the Presence of a Digital Platform}

Depending on whether the platform targets only the frequent consumers or caters to all of them, two additional scenarios emerge; henceforth, we will call them as Cases 4 and 5. Before we discuss them in detail, the following result is necessary:

LEMмA 4. If it allows the platform to enter the market, the theater would have no incentive to offer a competing pass of its own.
The intuition behind Lemma 4 is as follows. When it wants to block the platform's entry, the theater can only do so by jacking up the price above $\sqrt{2 \delta}$. However, as mentioned above, this maneuver may be costly as it may result in a disproportionate loss in demand. However, if the theater has the necessary technology to offer a subscription pass of its own, it can mitigate some of the loss by extending an effective price reduction through this pass to its subscribers. This way, its ability to offer its own pass grants the theater a cheaper option to block the entry if it so desires. Naturally, when it is not optimal to stave off the entrant, the option its own pass creates is no longer of any value, and the theater finds no incentive to continue offering its own pass.

Lemma 4 tells us that, in a subgame-perfect equilibrium, only one pass can exist in the market, not two. It also tells us that, in Cases 4 and 5, the theater only sells individual tickets. Of course, as per Lemma 2, it would continue to collect $r=p$ from the platform every time a subscriber walks in to watch a movie.

Case 4: Digital Platform Targets the Frequent Consumers Only. In this case, the theater makes $(p-c)$ for each high-type moviegoer; the rest continues to buy individual tickets as before. Therefore, the theater's total profit can be written as:

$$
\pi= \begin{cases}\lambda n(p-c)+ & \\ n(p-c)(1-\lambda)(1-p-\mu), & \text { if } p<1-\mu, \\ \lambda n(p-c), & \text { otherwise. }\end{cases}
$$

Once again, the second case $(p \geq 1-\mu)$ arises when the low type is priced out of the market. The theater maximizes this profit subject to the platform's participation constraint, $p<\sqrt{2 \delta}$. The solution to this optimization problem can be expressed as:

Proposition 4. When the platform targets only the frequent consumers, the theater's optimal price is given by:

$p^{*}= \begin{cases}p_{4}^{*}=\frac{c-\mu}{2}+\frac{1}{2(1-\lambda)}, & \text { if } \lambda<\frac{1-(c+\mu)}{2-(c+\mu)} \text { and } \delta \geq \Delta_{1}, \\ p_{4 \mathrm{~A}}^{*}=\sqrt{2 \delta}, & \text { if } \delta<\Delta_{1} \text { and } \delta<\Delta_{2}, \\ p_{4 \mathrm{~B}}^{*}=\sqrt{2 \delta}, & \text { if } \lambda \geq \frac{1-(c+\mu)}{2-(c+\mu)} \text { and } \delta \geq \Delta_{2},\end{cases}$

where $\Delta_{1}=\frac{(1+(1-\lambda)(c-\mu))^{2}}{8(1-\lambda)^{2}}$ and $\Delta_{2}=\frac{(1-\mu)^{2}}{2}$ The profit is given by $\pi_{4}^{*}=\frac{n(1-(1-\lambda)(c+\mu))^{2}}{4(1-\lambda)}, \pi_{4 \mathrm{~A}}^{*}=$ $n(\sqrt{2 \delta}-c)(1-\mu-\sqrt{2 \delta}+\lambda(\sqrt{2 \delta}+\mu))$, or $\pi_{4 \mathrm{~B}}^{*}=$ $n \lambda(\sqrt{2 \delta}-c)$. 
The platform sets $g^{*}=n\left(\delta+p^{*}\left(1-\frac{p^{*}}{2}\right)\right)$. The platform makes zero profit in Cases $4 A$ and $4 B$, and makes a profit of $\pi_{4, \text { plat }}=n \lambda\left(\delta-\frac{(1+(1-\lambda)(c-\mu))^{2}}{8(1-\lambda)^{2}}\right)>$ 0 in Case 4.

It is important to note that the profit function above is linear in $p$ for $p \geq 1-\mu$, and an interior solution is not possible there. Therefore, an interior solution can happen only for $p<1-\mu$, implying that $p_{4}^{*}$ must be less than $(1-\mu)$. This, in turn, means that both segments are served in Region 4 . The two limit regions, $4 \mathrm{~A}$ and $4 \mathrm{~B}$, are quite interesting. In these regions, the interior $p^{*}$ is so high that, as such, the platform would not enter the market. However, in these two cases, upon finding the platform's presence beneficial, the theater simply holds the price of individual tickets infinitesimally below the limit price of $\sqrt{2 \delta}$, enticing the platform to barely enter the market. The difference between the two cases is that, in $4 \mathrm{~A}$, the theater serves the infrequent segment, that is, $p_{4 \mathrm{~A}}^{*}=\sqrt{2 \delta}<1-\mu$, but in $4 \mathrm{~B}, p_{4 \mathrm{~B}}^{*}=$ $\sqrt{2 \delta} \geq 1-\mu$ and the infrequent segment is effectively priced out.

Case 5: Digital Platform Caters to All Consumers. In this case, the theater makes $(p-c)$ every time a moviegoer walks in, and no one buys individual tickets any longer. Therefore, the theater's total profit can be written as:

$$
\pi=\lambda n(p-c)(\lambda+(1-\lambda)(1-\mu)) .
$$

It is clear that this profit is linearly increasing in $p$, and there can be no interior solution as such. Therefore, for this case to occur, the theater must set $p$ in such a way that it becomes possible for the platform to offer the pass to both the segments, with no incentive to price the infrequent segment out of the market. It may seem from Theorem 2 that the theater can make it barely possible for the platform to cater to all consumers by setting a $p$ that satisfies $\delta=\frac{p(p+2 \lambda \mu)}{2(1-\mu)}$, implying $p=\sqrt{2 \delta(1-\mu)+(\lambda \mu)^{2}}-\lambda \mu$. For this to occur, though, the platform must also play along and set $g=n\left(\delta(1-\mu)+p\left(1-\mu-\frac{p}{2}\right)\right)$. However, in that case, the platform would make no profit at all and, as it turns out, the platform can actually do better. This it can do by setting $g=$ $n\left(\delta+p\left(1-\frac{p}{2}\right)\right)$ and attracting only the high type to the pass, thus violating the condition that both the segments be served.

Therefore, in order to induce the platform to cater to both the segments, the theater must reduce the price further to a level where $\delta=\frac{p(p(1-\lambda)+2 \lambda \mu)}{2(1-\lambda-\mu)}$, a point at which the platform becomes indifferent between the two choices.

Accordingly, the equilibrium can be stated as:
Proposition 5. When the platform caters to all consumers, in equilibrium, the theater charges a price of $p_{5}^{*}=\sqrt{\frac{2 \delta(1-\lambda-\mu)}{1-\lambda}+\left(\frac{\lambda \mu}{1-\lambda}\right)^{2}}-\frac{\lambda \mu}{1-\lambda}$ and makes a profit of $\pi_{5}^{*}=n(1-\mu(1-\lambda))\left(p_{5}^{*}-c\right)$. The platform sets $g_{5}^{*}=n\left(\delta(1-\mu)+p_{5}^{*}\left(1-\mu-\frac{p_{5}^{*}}{2}\right)\right)$ and makes a profit of $\pi_{5, \text { plat }}^{*}=\frac{n \lambda \mu\left(\delta+\lambda p_{5}^{*}\right)}{1-\lambda}$.

\subsection{Theater's Optimal Reaction Facing Entry of a Digital Platform}

It is clear from this analysis that the theater can adopt any one of the above five strategies, implying that the equilibrium could occur in any one of the nine cases discussed earlier (that is, Cases 15 and the four limit cases). Naturally, the theater would choose the strategy that yields the highest profit for a given set of parameter values. Since closed-form profit expressions exist in all the nine cases - see Propositions 1-5-we can analytically compare them in a pairwise fashion to obtain the $36\left(=\frac{9 !}{2 !(9-2) !}\right)$ boundaries that separate these nine cases. Similar to Theorem 1, closed-form expressions exist for all these boundaries, and the entire parameter space can be partitioned into appropriate regions. We illustrate the partitions in Figure 1; please note that not all nine cases necessarily occur in the equilibrium.

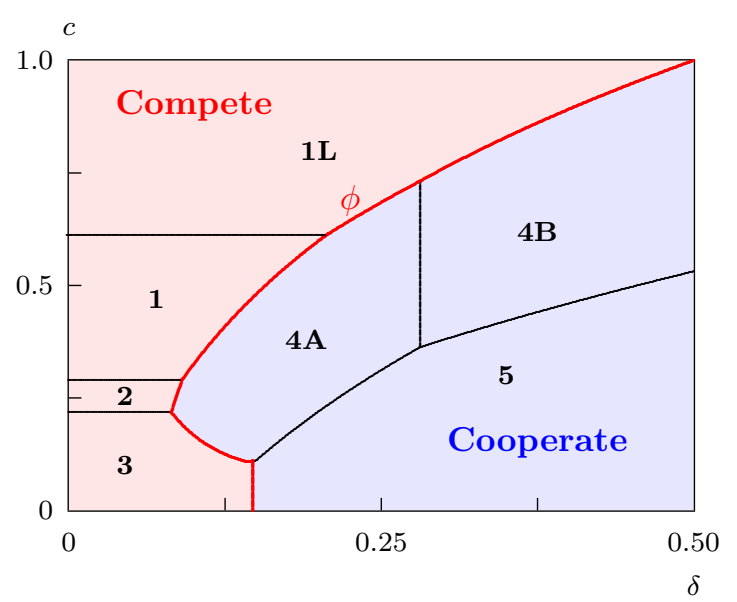

Figure 1. Partitions of the $(\delta, c)$ Space for Theater Having its Own Technology; $\lambda=0.3, \mu=0.25$

There are several interesting observations that can be made from this analysis. First, for a given $c$, the theater allows the platform to coexist only if it is more profitable to do so; we find that this actually happens when the platform brings in a sufficiently high $\delta$. When $\delta$ is small, the theater's profit does not change with $\delta$ (Regions 1, 1L, 2, 2L, 3). However, in 
Regions 4, 4A, 4B, 5-that is, when $\delta$ is larger-the theater's profit starts increasing with $\delta$.

Viewed differently, with respect to $\delta$, there is a threshold of resistance around which the theater's strategic response to the platform's entry shows a remarkable reversal; this threshold is denoted $\phi$ and marked by the red curve in Figure 1 . When $\delta$ is to the left of $\phi$, the benefit to the theater is not adequate and the theater sees no reason to cooperate with the platform, so the theater resists the entry. As $\delta$ moves to the right of the $\phi$ curve, though, the platform's appeal grows and it becomes difficult for the theater to keep it out of the market. Initially, in Regions $4 \mathrm{~A}$ and $4 \mathrm{~B}$, the theater accommodates the platform while raising $p$ to wrest away all of the platform's profits. In other words, the theater effectively holds off the platform - although the platform enters the market, it barely treads water, as the theater usurps any extra value generated by the platform's superior product.

Nevertheless, as $\delta$ gets larger still, the theater realizes that holding off the platform is now much costlier, as a high $p$ starts taking a heavy toll on the overall demand. A point is finally reached where, in fact, it becomes profitable for the theater to back off, causing the equilibrium to shift to Region 4 or 5 . Viewed another way, the theater can marginalize the platform when $c$ is moderate (Regions $4 \mathrm{~A}$ and $4 \mathrm{~B}$ ). However, if $c$ is small, setting $p$ too high and forgoing a substantial portion of the market becomes counterproductive. So, the theater fully accommodates the platform and allows it to walk away with some profit (Regions 4 and 5).

In broader terms, the intermediary is a middleman, and resisting its entry to a point indeed makes good economic sense. However, resisting becomes difficult in certain situations, particularly if doing so requires raising the ticket price to a level that is disproportionately high when compared to $c$. So, if $\delta$ is high, sharing the additional value generated is preferable to costly resistance.

\section{4. "Hot-and-Cold" Relationship}

Why did AMC partner with MoviePass in the first place, only to break the alliance in less than three years, and why did MoviePass offer a pricing scheme that is not sustainable for long? Our modeling experiment provides a plausible economic rationale behind such apparently anomalous actions.

To understand, we must note that the equilibrium discussed in Figure 1 is derived under the assumption that the theater has the necessary IT infrastructure for offering its own subscription service electronically. If it does not, Cases $2,2 \mathrm{~L}$, and 3 are no longer possible, as the theater now lacks the ability to offer its own pass. The resulting equilibrium is obtained in a manner similar to the one adopted earlier. We simply compare the theater's profits in Cases 1, 1L, 4, 4A, 4B, and 5, and pick the case that results in the maximum for a particular set of parameter values. The resulting equilibrium is illustrated in Figure 2. In this figure, the boundary between

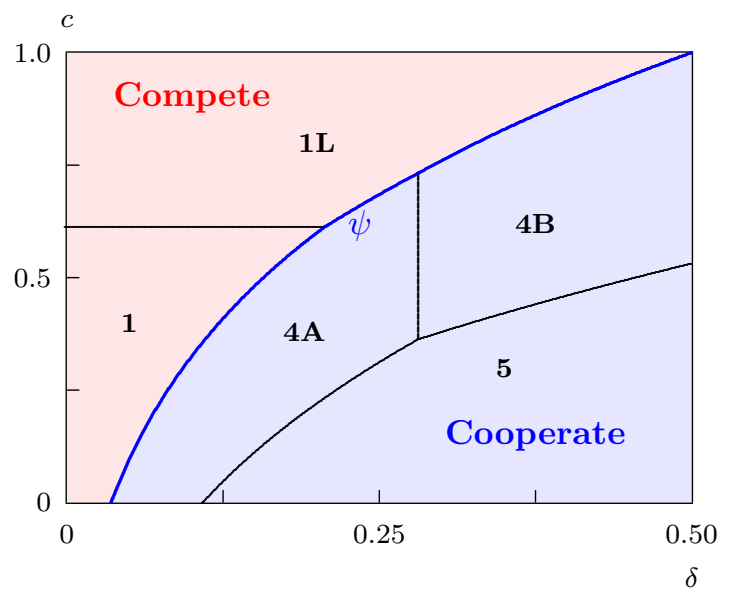

Figure 2. Partitions of the $(\delta, c)$ Space for Theater without Technology; $\lambda=0.3, \mu=0.25$

where the theater resists the platform and where they coexist is denoted $\psi$ and is marked by a blue curve representing the new threshold of resistance. Comparing Figure 2 with Figure 1, one can see that, as long as $c$ is not too large, the blue boundary, $\psi$, is to the left of the red one, $\phi$. Viewed differently, there is a swing region where, depending on whether or not it possesses the required technical infrastructure, the theater's response could be markedly different. In order to see this region more clearly, in Figure 3, we superimpose Figure 2 on Figure 1, and zoom into the interesting portion. This way, the swing region is clearly demarcated by the dashed blue and red curves.

Now, consider a situation where the real-world context is within this region and, say, is represented by the purple dot. If that is indeed the case, the theater's strategy would encounter a remarkable shift depending on whether it has the required technology or not. If it does not, the theater, being to the right of the blue curve $(\psi)$, would tend to accommodate the platform. On the other hand, when the theater acquires the necessary technology, the red curve $(\phi)$ takes over; being to the left of that curve, the theater now competes fiercely with the platform and forces it out of the market. What is really interesting is that, irrespective of the values of $\lambda$ and $\mu$, a portion 


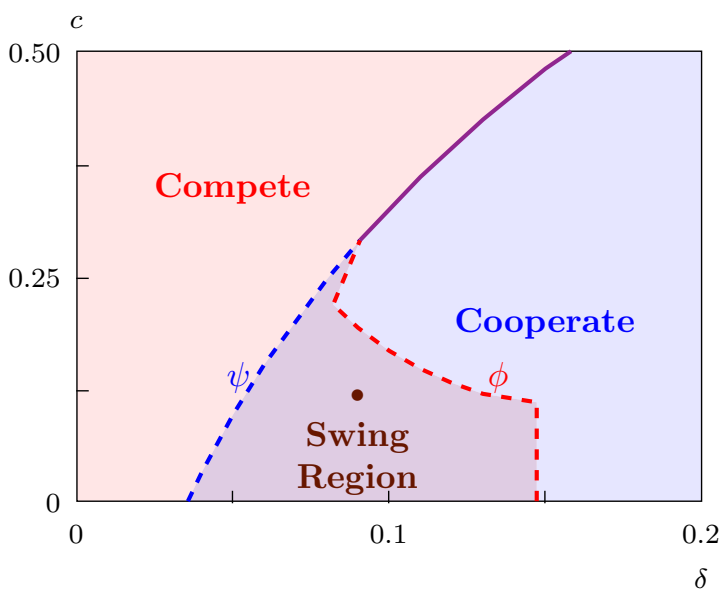

Figure 3. Shifting Behavior in the $(\delta, c)$ Space; $\lambda=0.3$, $\mu=0.25$

of the blue curve is always to the left of the red one as long as $c$ is not too large, implying that the swing region always exists.

Theorem 3. For all values of $\lambda, \mu \in(0,1)$, there is always a non-empty swing region, where the theater cooperates when it does not have the necessary technology, but competes when it acquires the technology.

According to Theorem 3, the swing region always exists, and this region might explain what we all observed in practice. Towards the end of 2014, AMC was just warming up to the idea of having a subscription model in place but did not have the necessary technology. ${ }^{2}$ Therefore, its initial support for MoviePass' subscription platform to fill a few more empty seats - or, perhaps, to learn from MoviePass' platform by watching it from close quarters - was a sensible strategy at that time. However, AMC must have known all along that it could make a better profit if it possessed its own technology, so it must have invested in developing a platform of its own. A little more than two years later, likely by the middle of 2017, AMC had the basic system in place, and its initial strategy was no longer the right one. It is at this point that AMC decided to adopt an aggressive posture. In other words, our exercise finds that the real-world events have a possible economic basisAMC knew what it was doing both in 2014 and in

\footnotetext{
${ }^{2}$ Interestingly, even though AMC now has the basic technology necessary to administer its subscription service over the smartphone, it still lags behind MoviePass in several regards. For example, it does not integrate the smartphone's GPS information and cannot track its subscribers' location on a real-time basis. It also does not have the ability to push targeted advertisement based on the customer's location.
}

2017, and its behavior is in line with that of a profitmaximizing economic agent.

Now, what can we say about the actions taken by MoviePass? If our economic model is to be believed, even back in 2014, MoviePass was likely aware of the entire situation that would unfold once AMC developed its own infrastructure. In other words, as a rational economic agent, MoviePass could anticipate that AMC would eventually break the alliance. The only way MoviePass could get any cooperation from AMC had to be by increasing the perceived value of $\delta$ and moving the purple point to the right of the red boundary in Figure 3. In fact, the $\$ 9.95$ subscription fee - a pricing strategy that was compared to burning money by many - was likely a move geared towards just that. Within a year of announcing the deal, MoviePass' subscriber base ballooned to over three millions, making it difficult for theater chains to ignore MoviePass [3, 6]. Penetration pricing to gain market share has been a well-known strategy [12, 15], and MoviePass' introductory pricing was certainly no different. Those who speculated that MoviePass would go bankrupt had somehow assumed implicitly that MoviePass would continue to offer this deal for ever. However, such introductory pricing is usually for a limited period. It is, therefore, no coincidence that this pricing deal has recently been abandoned in favor of a new one [13]. ${ }^{3}$

How can a bigger subscriber base enhance $\delta$, the value added by the platform? The answer lies in the fact that, after all, MoviePass is a digital platform bringing different theater chains and their consumers together. Two-sided markets are known to generate network effects, both within as well as across the two sides [16]. The cross-platform network effect is easy to see. As more consumers flock to the platform, it becomes difficult for theater chains to ignore that segment, creating an economic incentive for more of them to join in. When a moviegoer sees more theaters in his choice list, it gives him more flexibility in terms of where he may watch the movie, resulting in a higher $\delta$. Therefore, as MoviePass increases its subscription base, it also increases the perceived $\delta$.

To understand the same-side network effect, we must note that MoviePass can amass a significant volume of data on its subscribers' viewing and other consumption habits, along with location and movement data. In fact, just to use the MoviePass app - the only way one can access the platformpermission must be granted to use the location

${ }^{3}$ Effective August 15, 2018, MoviePass allows only three free movies per month for a monthly fee of $\$ 9.95$; it also imposes certain restrictions on the choice of movies and theaters. 
service of the device, at least partially (that is, when the app is on). Running this huge volume of data through sophisticated data analytics tools, MoviePass can mine useful insights on personalization, recommendation, market segmentation, and product promotion. This, in turn, can not only enhance the platform's value to its subscribers, but can also bring a separate value proposition to theaters and studios. MoviePass can also make alliances with other types of merchants-bars, restaurants, coffee shops, flower and gift shops-and offer discounts on their products, creating positive crossconsumption effects. For this part, MoviePass can target businesses with whom MoviePass shares common consumers; this way, MoviePass can make further use of the insights it learns from data analytics to demonstrate that it can indeed influence subscribers' consumption behavior through appropriate marketing maneuvers [2].

Not surprisingly, MoviePass is already doing all of the above. HMA, the data analytics firm that currently owns the majority share in MoviePass made the acquisition simply to get access to the platform that stores, among other things, its subscribers' consumption behavior. MoviePass itself has openly boasted about its capability to mine the huge volumes of subscriber data that it has amassed. For example, at this point, MoviePass accounts for about only $3 \%$ of all box office sales; however, the number apparently jumps to $10 \%$ when MoviePass actively pushes a product [2]. Clearly, the main thrust at MoviePass seems to be mining deeper insights about consumer behavior and monetizing these insights in an innovative way. Naturally, many of these insights may also allow MoviePass to create better value for its subscribers, thereby influencing their perception of $\delta$ positively. For example, features such as timely recommendation and notifications, targeted discounts, and access to consumer reviews could enhance a subscriber's overall experience with MoviePass. Naturally, the usefulness of such features - and their impact on $\delta$ - ought to depend on the quality and quantity of such insights, which in turn should depend on the size of the subscription base that generates the underlying data.

\section{Conclusions}

Motivated by the recent feud between a theater giant (AMC) and a new digital platform (MoviePass), we investigate their strategic interactions in a gametheoretic setting. A key feature of this setting is that the theater - the seller - has the option of blocking the platform, a nonessential middleman. And, somewhat unconventionally, the theater must set a high individual ticket price to do so. Since a high price can adversely affect the demand, the theater deters the entry only when the benefit of cutting out the middleman is large vis-à-vis the loss from a declining demand. The resulting equilibrium depends on the model parameters, in particular how the extra value offered by the middleman $(\delta)$ compares with the marginal cost to the theater $(c)$. When the marginal cost is low and the extra value is high, the theater accommodates the entry; conversely, when the cost is high and the value low, the theater blocks the entry.

Interestingly, the theater's ability to offer a pass of its own also affects its ability to deter the middleman. The pass actually allows the theater to mitigate the adverse effect of a higher price necessary to deter the entry, as the theater can now extend an effective price reduction via the pass to certain portions of the market. Therefore, the ability to offer a pass also translates to an enhanced ability to deter the entry of the middleman. Since AMC did not have a subscription platform of its own initially but developed one later, its incentives to accommodate MoviePass also waned subsequently. In other words, insights from our model point to the plausible economic rationale for the "Hot-and-Cold" relationship between AMC and MoviePass in recent years. More importantly, they also point to the fact that a theater should view investments into building a subscription platform of its own from the perspective of a strategic deterrent, and not merely as a tool to support routine business operations.

Although developed within the context of the movie industry, our insights may be applicable to other industries where third-party subscription services are beginning to emerge. For example, Gympass offers a flexible pass that allows its subscribers to access up to thirty thousand gyms across several metropolitan areas in the USA. Similar to MoviePass, Gympass charges its subscribers a monthly fee and, in exchange, promises them the flexibility to workout wherever and whenever. ClassPass, which is similar, allows its subscribers to choose any fitness class anywhere with just one subscription. ClassPass has presence in several major US cities. Another recent startup known as Slay is seeking to invade the beauty salon market with a very similar approach. Likewise, MealPal is offering gourmet lunch at participating restaurants as a subscription service. While each of these contexts has its idiosyncrasies, the broad insights gleaned from our model may shed some light on them as well. 


\section{References}

[1] M. Armstrong, "Price Discrimination by a ManyProduct Firm," Review of Economic Studies, 1999 (66:1), pp. 151-168.

[2] B. Barrett, "How Does MoviePass Make Money? We're Starting to Find Out," Wired, 2018 (January 26, 2018, https://www.wired.com/story/ moviepass-second-act/. Accessed Aug 27, 2018.

[3] K. Canales and J. Cheng, "MoviePass had a year of meteoric subscriber growth and that's a problem," Business Insider, 2018 (July 31, 2018, https://www.businessinsider.com/moviepass3-million-subscriber-count-isnt-helping-charts2018-7. Accessed Aug 30, 2018.

[4] A. Chircu and R. Kauffman, "Strategies for Internet Middlemen in the Intermediation/Disintermediation/Reintermediation Cycle," Electronic Markets, 1999 (9:1-2), pp. 109-117.

[5] —, "Reintermediation Strategies in Businessto-Business Electronic Commerce," International Journal of Electronic Commerce, 2000 (4:4), pp. 742.

[6] A. Chow, "Tracking MoviePass's Bumpy History as It Slashes Movie Allowance," The New York Times, 2018 (July 31, 2018, https://www. nytimes.com/2018/07/31/movies/moviepasstimeline.html. Accessed Aug 7, 2018.

[7] E. Clemons, S. Reddi, and M. Row, "The Impact of Information Technology on the Organization of Economic Activity: The 'Move to the Middle' Hypothesis," Journal of Management Information Systems, 1993 (10:2), pp. 9-35.

[8] J. Disis, "Is the end near for MoviePass?," CNN, https://money.cnn.com/2018/05/10/media/ moviepass-how-long-can-it-last/index.html. 2018. Accessed Sep 14, 2018.

[9] T. Hendershott and J. Zhang, "A model of direct and intermediated sales," Journal of Economics \& Management Strategy, 2006 (15:2), pp. 279-316.

[10] C. Hess and C. Kemerer, "Computerized Loan Origination Systems: An Industry Case Study of the Electronic Markets Hypothesis," MIS Quarterly, 1994 (18:3), pp. 251-275.

[11] L. Hitt and P. Chen, "Bundling with Customer SelfSelection: A Simple Approach to Bundling LowMarginal-Cost Goods," Management Science, 2005 (51:10), pp. 1481-1493.

[12] R. Holden and T. Nagle, "Kamikaze Pricing," Marketing Management, 1998 (7:2), pp. 30-39.

[13] S. Mendelson, "The New MoviePass Plan Is A Disaster For Smaller Movies And Indie Flicks," Forbes,
2018 (August 6, 2018, https://www.forbes.com/ sites/scottmendelson/2018/08/06/the-new-movie pass-plan-is-a-disaster-for-theaters-and-smallmovies/\#73933d714336. Accessed Oct 5, 2018.

[14] MPAA, "2017 MPAA Theme Report," Motion Pictures Association of America, https://www. mpaa.org/wp-content/uploads/2018/04/MPAATHEME-Report-2017_Final.pdf. 2017. Accessed Jul 8, 2018.

[15] P. Noble and T. Gruca, "Industrial Pricing: Theory and Managerial Practice," Marketing Science, 1999 (18:3), pp. 435-454.

[16] G. Parker and M. Van Alstyne, "Two-Sided Network Effects: A Theory of Information Product Design," Management Science, 2005 (51:10), pp. 1494-1504.

[17] J. Strick, "The Economics of the Motion Picture Industry: A Survey," Philosophy of the Social Sciences, 1978 (8:4), pp. 406-417.

[18] A. Sundararajan, "Managing Digital Piracy: Pricing and Protection," Information Systems Research, 2004 (15:3), pp. 287-308.

[19] J. Tirole, The Theory of Industrial Organization, The MIT Press, 1992. 